\title{
Consumo alimentar e estado nutricional de crianças atendidas em serviços públicos de saúde do município de Viçosa, Minas Gerais
}

\section{Food consumption and nutritional profile of children}

seen in public health services of Viçosa, Minas Gerais, Brazil

\author{
Ana Augusta Monteiro CAVALCANTE ${ }^{1}$ \\ Adelson Luiz Araújo TINÔCO ${ }^{1}$ \\ Rosângela Minardi Mitre COTTA ${ }^{1}$ \\ Rita de Cássia Lanes RIBEIRO ${ }^{1}$ \\ Conceição Angelina dos Santos PEREIRA ${ }^{1}$ \\ Sylvia do Carmo Castro FRANCESCHINI ${ }^{1}$
}

\section{RES U M O}

\section{Objetivo}

Avaliar o consumo alimentar e o estado nutricional de 174 crianças, entre 12 e 35 meses de idade, atendidas na rede pública de saúde de Viçosa, Minas Gerais, Brasil.

\section{Métodos}

O consumo alimentar foi investigado por meio de dois inquéritos dietéticos (recordatório 24 horas e questionário de freqüência de consumo alimentar). O estado nutricional foi avaliado pelos índices antropométricos peso/idade, peso/estatura e estatura/idade, e associado ao consumo alimentar. Utilizou-se o Teste " $t$ " Student para comparar médias, Teste $\chi^{2}$ para verificar associação entre variáveis e Odds Ratio para avaliar fatores de risco envolvidos na ocorrência de déficits nutricionais $(p<0,05)$.

\section{Resultados}

A quantidade média de energia consumida excedeu a recomendação para ambos os sexos $(p<0,0001)$. A ingestão habitual de energia e nutrientes foi estatisticamente significante e maior entre os meninos $(p<0,0001)$, exceto para vitamina C. Prevalências de inadequação foram observadas para as vitaminas C e A $(96,6 \%$ e $36,8 \%$ ) e os minerais ferro e zinco (13,2\% e 99,4\%). Com relação ao estado nutricional, as prevalências de desnutrição encontradas foram 13,2\% para o índice peso/idade, 13,8\% para peso/estatura e 4,0\% para estatura/idade. Encontrou-se associação positiva entre déficit nutricional e consumo alimentar inadequado.

\footnotetext{
1 Programa de Pós-Graduação, Departamento de Nutrição e Saúde, Universidade Federal de Viçosa. Av. P.H. Rolfs, s/n, 36570-000, Viçosa, MG, Brasil. Correspondência para/Correspondence to: A.A.M. CAVALCANTE. E-mail: <monteirocavalcante@yahoo.com.br>.
} 


\section{Conclusão}

Estes resultados demonstram que o consumo alimentar inadequado tem contribuído para os déficits nutricionais do grupo pré-escolar. Recomenda-se a realização continuada de estudos de consumo alimentar nessa população, para conhecer sua prática alimentar.

Termos de indexação: antropometria; criança; consumo de alimentos; estado nutricional.

\section{A B S T R A C T}

\section{Objective}

To assess the food consumption and the nutritional status of 174 children, ages between 12 and 35 months seen in the public health services of the municipality of Viçosa, Minas Gerais, Brazil.

\section{Methods}

Two questionnaires were applied to collect the dietary data (24-hour recall and food frequency questionnaire). The nutritional status was determined by using anthropometric indices of weight/age, weight/stature and stature/age, and associated with food consumption. The Student's " $t$ " test was used for comparing means, the chi-square test to verify associations between variables and the Odds Ratio to determine the risk factors involved in the occurrence of nutritional deficiencies $(p<0.05)$.

\section{Results}

The medium amount of energy consumed exceeded the recommendation for both genders $(p<0.0001)$. The usual intake of energy and nutrients was statistically significant and higher for boys than for girls $(p<0.0001)$ exception being made for vitamin C. Inadequacy prevalence was observed for the vitamins A and C (96.6\% and $36.8 \%)$ and the minerals iron and zinc (13.2\% and 99.4\%). Malnutrition prevalence was found for weight/age (13.2\%), weight/stature (13.8\%) and stature/age (4.0\%). Positive association was found between nutritional deficiencies and food consumption inadequacy.

\section{Conclusion}

The results showed that inadequate food consumption contributes to deficiencies in the nutritional status of the preschool group. Further and continued studies to evaluate the food consumption of preschool children are recommended, including research into the feeding practices in that age group and in the overall population.

Indexing terms: anthropometry; child; food consumption; nutritional status.

\section{N T R O D U Ç Ã O}

A complexidade da dieta humana tem instigado pesquisadores a procurar os meios mais adequados para avaliar qualitativa e quantitativamente o consumo de alimentos, dimensionar a adequação de nutrientes e estudar a relação entre dieta e doença'.

Estudos sobre consumo alimentar de crianças brasileiras são escassos. Embora o quadro nutricional já revele mudanças no padrão nutricional, indicando a coexistência de desnutrição e de sobrepeso e obesidade em todos os segmentos da população, a desnutrição infantil ainda constitui um grave problema de saúde pública no país. Alguns estudos mostram que, embora de natureza multifatorial, os hábitos alimentares inadequados estão entre os fatores determinantes que mais repercutem desfavoravelmente sobre o estado nutricional das crianças, particularmente nas áreas econômica e socialmente mais desfavorecidas ${ }^{2-5}$.

Em artigo de revisão abrangente, incluindo crianças brasileiras, Post et al. ${ }^{6}$ mostraram prevalências de $20,6 \%, 8,2 \%$ e $2,4 \%$ em déficits de estatura para a idade, de peso para a idade e de peso para a estatura, respectivamente.

Alguns autores ressaltam a importância de conhecer a prática alimentar de crianças, antes da formulação e implementação de qualquer programa de intervenção nutricional, para 
avaliação adequada do impacto da medida implementada ${ }^{7,8}$.

As pesquisas de consumo alimentar constituem instrumentos eficazes e de baixo custo, que permitem a obtenção de informações sobre as características da ingestão dietética de grande parte da população e podem ser realizadas por meio de inquéritos dietéticos, que determinam, de forma indireta, o estado nutricional de grupos ou indivíduos. Além disso, apresentam como principal vantagem, a possibilidade de mensuração do consumo de alimentos, o que proporciona condições para inferir, com maior precisão, a quantidade de alimentos efetivamente consumida por famílias ou mesmo indivíduos ${ }^{8-10}$.

Considerando a importância da alimentação na promoção da saúde e nutrição das crianças e o crescente interesse na relação entre dieta e ocorrência de distúrbios nutricionais, este estudo teve como objetivo caracterizar o consumo alimentar e o estado nutricional de crianças de 12 a 35 meses de idade, atendidas na rede pública de saúde do município de Viçosa, Minas Gerais.

\section{MÉ T O D O S}

Trata-se de um estudo epidemiológico nutricional, de corte transversal, pelo qual foram avaliados o consumo alimentar e o estado nutricional de 174 crianças de 12 a 35 meses de idade, atendidas na rede pública de saúde do município de Viçosa, Minas Gerais. A pesquisa foi apresentada nos serviços de saúde aos profissionais de saúde e às mães ou responsáveis, que permitiram a participação das crianças, por meio de assinatura de um termo de Consentimento Livre e Esclarecido.

A coleta de dados foi realizada no período de outubro/2003 a janeiro/2004, por meio de entrevistas com as mães ou responsáveis para preenchimento de questionário previamente testado, contendo informações sobre inquérito dietético e identificação da situação socioeconômica, demográfica e de assistência à saúde. O consumo alimentar foi obtido pela aplicação, uma única vez, de dois inquéritos dietéticos: o questionário de freqüência de consumo alimentar e o recordatório 24 horas.

O Questionário de Freqüência Alimentar (QFCA) utilizado neste estudo foi quantitativo, e adaptado a partir de um questionário desenvolvido pelo Departamento de Nutrição e Saúde da Universidade Federal de Viçosa, o qual apresenta caráter regionalizado, validado para a população de Viçosa, Minas Gerais ${ }^{11}$.

O QFCA adaptado dispõe de 62 itens alimentares usualmente presentes no hábito alimentar da região. Foram estabelecidas 11 unidades de tempo como categorias de resposta à freqüência do consumo alimentar, sendo estas: 1 a 7 correspondendo a quantos dias na semana efetuou-se o consumo. Além das opções T, Q, R e $\mathrm{N}$, correspondendo, respectivamente, a: três vezes ao mês, quinzenalmente, raramente ou nunca, . Para quantificar o consumo, o questionário também dispõe da opção medida caseira em gramas ou mililitros, descrevendo a quantidade das porções consumidas. As medidas caseiras foram transformadas em gramas ou mililitros, com o auxilio de uma tabela apropriada para este fim ${ }^{12}$.

Para obter um padrão diário de ingestão de cada alimento contido no QFCA estabeleceram-se constantes para cada dia de ingestão. Assim, em relação a uma semana, obtiveram-se as seguintes constantes: $1 \mathrm{dia}=0,14(1 \div 7)$; 2 dias $=0,28(2 \div 7) ; 3$ dias $=0,42(3 \div 7) ; 4$ dias $=0,57$ $(4 \div 7) ; 5$ dias $=0,71(5 \div 7) ; 6$ dias $=0,85(6 \div 7) ; 7$ dias $=1(7 \div 7) ; 3 \times$ mês $=0,1(3 \div 30) ; 2 \times$ mês $=0,06$ $(2 \div 30)$. As opções raramente e nunca não foram consideradas como ingestão ${ }^{13}$.

Após a realização dos cálculos (multiplicando-se a constante encontrada pela medida caseira do alimento considerado), obteve-se a ingestão de um dia, ou seja, a contribuição usual de cada alimento na ingestão alimentar das crianças estudadas. Com esses dados construiu-se o QFCA no software Diet PRO (versão 4.0).

Com a soma obtida das médias dos dois inquéritos dietéticos utilizados neste estudo, 
obteve-se a média de ingestão habitual das crianças pesquisadas.

O Questionário de Freqüência de Consumo Alimentar tem se mostrado capaz de caracterizar a dieta habitual de indivíduos numa única aplicação, avaliar a ingestão alimentar de populações, ter boa reprodutibilidade e validade aceitável, relacionar a dieta à ocorrência de doença, ser mais prático, informativo, de fácil aplicação e de baixo custo $8,14,15$.

O método recordatório 24 horas é um instrumento de avaliação da ingestão de alimentos e nutrientes de indivíduos e grupos populacionais. É um instrumento validado, freqüentemente utilizado em estudos epidemiológicos, capaz de avaliar a ingestão atual de indivíduos, em geral bem aceito pelos entrevistados, de baixo custo e não demanda muito tempo na aplicação, mas requer um nutricionista ou entrevistador bem treinado para a realização de coleta de dados ${ }^{16}$.

A aplicação do método recordatório 24 horas consiste em obter informações escritas ou verbais sobre a ingestão alimentar das últimas 24 horas, com dados sobre os alimentos atualmente consumidos e informações sobre peso/tamanho das porções, que deveriam ser, em tese, fornecidas com o auxílio de fotografias ou modelos de porções ${ }^{8}$.

As informações dos alimentos consumidos foram anotadas seguindo a ordem das refeições principais, intercaladas pelos lanches, incluindo a primeira até a última refeição. Registraram-se o tipo de alimento, a quantidade, a forma de preparação, bem como a hora do consumo. Após registrados em medidas caseiras, os alimentos foram transformados em gramas ou mililitros, com o auxílio de uma tabela para avaliação de consumo alimentar apropriada para esse fim ${ }^{12}$.

Um álbum composto de desenhos de alimentos nas três dimensões normais - pequena, média e grande - e utensílios e medidas-padrão foram utilizados como recursos, para auxiliar o entrevistado a recordar a porção do alimento servido à criança e aumentar a confiabilidade das informações fornecidas ${ }^{17}$.
Os cálculos para quantificar o consumo de energia, proteína, carboidrato, lipídio, ferro, vitaminas $A$ e $C$ foram realizados com o auxílio do software DietPRO4 versão 4.0. A avaliação da ingestão dietética foi realizada com base nas Ingestões Dietéticas de Referência (Dietary Reference Intakes - DRI). As DRI são valores de referência de ingestão de nutrientes que reúnem conceitos e conhecimentos científicos mais atualizados e, basicamente, são utilizadas no planejamento e avaliação das dietas, estimando a ingestão alimentar de indivíduos e de grupos populacionais ${ }^{18-20}$.

O peso das crianças com idade inferior a 24 meses foi aferido em balança eletrônica digital infantil, com capacidade máxima de $15 \mathrm{~kg}$ e sensibilidade de $5 \mathrm{~g}$. Para aquelas com idade igual ou maior que 24 meses, utilizou-se balança com capacidade máxima de $150 \mathrm{~kg}$ e precisão de $100 \mathrm{~g}$. O comprimento foi obtido utilizando-se antropômetro infantil de madeira, do tipo horizontal, com régua graduada em centímetros e subdividida em milímetros, com extensão de 1,5 metros. A estatura foi verificada com fita métrica metálica (Microtoiose - Stanley Ltda/CMS Weighting Equipment Ltd) com extensão de 2,0 metros, dividida em centímetros e subdividida em milímetros, com visor plástico e esquadro acoplado em uma das extremidades.

As crianças foram pesadas e medidas segundo técnicas preconizadas por Jelliffe, em $1966^{21}$. Os índices antropométricos P/E (peso/ estatura), E/I (estatura/idade) e P/I (peso/idade) foram adotados para avaliar o estado nutricional das crianças e foram expressos em escore- $Z$ ou unidades de desvio-padrão de afastamento da mediana da população de referência do Center for Disease Control and Prevention/National Center for Health Statistics, considerando-se: desnutrição ( $<-2$ escore-Z), risco nutricional $(\geq-2$ e $<-1)$ e eutrofia $(\geq-1$ a $\leq+1)$. Especificamente para os índices P/I e P/E considerou-se: risco de sobrepeso $(>+1$ até $\leq+2)$ e sobrepeso $(>+2)$. Para o índice $E / /$ valores $>-1$ escore-Z foram considerados como eutrofia 22 . 
$\mathrm{Na}$ análise descritiva dos dados de consumo alimentar foram calculadas: média, desvio-padrão, mediana, mínimo e máximo. Por meio do Teste " $t$ " Student foram verificadas as diferenças entre as médias do consumo de energia e nutrientes, segundo sexo. Para analisar as associações entre as variáveis empregou-se o teste Qui-quadrado. Calculou-se a Odds Ratio (Razão de (hances), para avaliar os fatores de risco envolvidos na ocorrência de déficits nutricionais entre as crianças do estudo. Os cálculos estatísticos foram realizados com o auxílio do programa Epi Info versão 6.04. O nível de rejeição da hipótese de nulidade foi 0,05 ou $5 \%$.

\section{RESULTADOS E DIS CUSS Ã O}

As análises descritivas de consumo habitual de energia, micro e macronutrientes, estimadas pela média dos inquéritos dietéticos (Tabela 1), mostraram consumo de energia e nutrientes superior, com diferenças estatisticamente significantes entre meninos $(p<0,05)$, quando comparado ao das meninas, exceto para a vitamina $\mathrm{C}$.

Analisando a necessidade estimada de energia observou-se que a média de ingestão de energia das crianças pesquisadas (Tabela 1) excedeu as necessidades calculadas, utilizandose as equações do Institute of Medicine ${ }^{23}$, sendo esta diferença estatisticamente significante entre os sexos, de acordo com as faixas etárias $(p<0,05)$.

Resultados semelhantes aos deste estudo foram encontrados em investigações ocorridas no México e nos Estados Unidos, que também utilizaram as novas equações do Institute of Medicine ${ }^{23}$, encontrando diferenças sempre

Tabela 1. Consumo habitual de energia, macro e micronutrientes estimado pela média de dois inquéritos dietéticos em crianças, segundo sexo. Viçosa, 2004.

\begin{tabular}{|c|c|c|c|c|c|c|c|c|c|}
\hline Nutrientes & Sexo & $n$ & Média & \pm & DP & Mediana & Mínimo & Máximo & Estatística \\
\hline \multirow[t]{3}{*}{ Energia (kcal) } & Masculino & 96 & 1058,04 & \pm & 326,50 & 978,50 & 482,00 & 1796,00 & \\
\hline & Feminino & 78 & 955,00 & \pm & 314,72 & 943,00 & 340,00 & 1710,00 & $p<0,0001$ * \\
\hline & Total & 174 & 1009,10 & \pm & 324,06 & 961,00 & 340,00 & 1796,00 & \\
\hline \multirow[t]{3}{*}{ Carboidrato (g) } & Masculino & 96 & 151,22 & \pm & 50,60 & 144,00 & 62,00 & 291,00 & \\
\hline & Feminino & 78 & 132,17 & \pm & 41,31 & 127,00 & 39,00 & 224,00 & $p<0,0001^{*}$ \\
\hline & Total & 174 & 142,69 & \pm & 47,50 & 133,50 & 39,00 & 291,00 & \\
\hline \multirow[t]{3}{*}{ Proteína (g) } & Masculino & 96 & 34,03 & \pm & 13,38 & 34,50 & 6,00 & 65,00 & \\
\hline & Feminino & 78 & 32,98 & \pm & 17,00 & 31,00 & 0,00 & 98,00 & $p<0,0001$ * \\
\hline & Total & 174 & 33,60 & \pm & 15,07 & 32,50 & 0,00 & 98,00 & \\
\hline \multirow[t]{3}{*}{ Lipídio (g) } & Masculino & 96 & 36,44 & \pm & 14,66 & 37,00 & 0,00 & 97,00 & \\
\hline & Feminino & 78 & 33,77 & \pm & 12,70 & 33,00 & 4,00 & 71,00 & $p<0,0001$ * \\
\hline & Total & 174 & 35,24 & \pm & 13,83 & 35,00 & 0,00 & 97,00 & \\
\hline \multirow[t]{3}{*}{ Vitamina A ( $\mu \mathrm{gER})$} & Masculino & 96 & 459,36 & \pm & 497,91 & 313,00 & 0,00 & 2987,00 & \\
\hline & Feminino & 78 & 319,21 & \pm & 311,81 & 236,50 & 0,00 & 1511,00 & $p<0,0001$ * \\
\hline & Total & 174 & 396,54 & \pm & 429,30 & 280,50 & 0,00 & 2987,00 & \\
\hline \multirow[t]{3}{*}{ Vitamina C (mg) } & Masculino & 96 & 2,72 & \pm & 17,00 & 0,00 & 0,00 & 163,00 & $p=0,116$ \\
\hline & Feminino & 78 & 1,92 & \pm & 10,04 & 0,00 & 0,00 & 81,00 & $p=0,090$ \\
\hline & Total & 174 & 2,36 & \pm & 14,27 & 0,00 & 0,00 & 163,00 & $p=0,028$ \\
\hline \multirow[t]{3}{*}{ Ferro (mg) } & Masculino & 96 & 5,94 & \pm & 2,66 & 6,00 & 2,00 & 14,00 & \\
\hline & Feminino & 78 & 4,91 & \pm & 2,47 & 4,00 & 0,00 & 14,00 & $p<0,0001 *$ \\
\hline & Total & 174 & 5,47 & \pm & 2,62 & 5,00 & 0,00 & 14,00 & \\
\hline
\end{tabular}

*Teste " $t$ " Student $p<0,05$ 
superiores entre os Requerimentos Estimados de Energia (EER) e a média de ingestão habitual de energia 24,25 .

A composição de macronutrientes da dieta, em termos de contribuição percentual dos macronutrientes, Acceptable Macronutrient Distribution Ranges (AMDR), com relação à energia proveniente das ingestões de lipídios, carboidratos e proteínas, foi calculada considerando o percentual de energia fornecido por cada um desses macronutrientes (30\% - 40\% para lipídio, 45\% - 65\% para carboidrato, 5\% - 20\% para proteína $)^{20}$.

Neste estudo, embora a proporção de crianças com ingestão de energia proveniente dos lipídios abaixo dos valores recomendados, tenha sido maior entre aquelas entre 12 a 23 meses de idade $(45,0 \%)$, se observou, nas duas faixas etárias (12 a 23 e 24 a 35 meses), uma elevada proporção de crianças abaixo do limite mínimo recomendado, $45,0 \%$ e $36,5 \%$, respectivamente.

Estes dados são importantes, particularmente com relação às faixas etárias estudadas, e evidenciam comprometimento no aporte de ácidos graxos essenciais dessas crianças. Os ácidos graxos, nos primeiros 18 meses de idade e, posteriormente, até os três anos de vida, desempenham papel fundamental na promoção do desenvolvimento físico e mental das crianças.

Ainda com relação à energia proveniente dos lipídios, se encontrou uma proporção relativamente elevada de crianças, em ambas as faixas etárias (12 a 23 e 24 a 35 meses de idade), consumindo acima do limite máximo permitido para lipídios pela AMDR, sendo 12,6\% e 15,7\%, respectivamente. Estes resultados, provavelmente, possam refletir uma introdução precoce e inadequada de alimentos da família. Observou-se, nas faixas etárias de 12 a 23 meses e 24 a 35 meses de idade, uma acentuada tendência de inadequação de energia proveniente dos lipídios, visto que cerca de $81,5 \%$ das crianças estudadas não alcançaram o limite mínimo da recomendação para esse macronutriente, que é 30,0\%.
Para carboidratos, uma grande proporção de crianças, em ambas as faixas etárias, 80,2\% e $87,3 \%$, permaneceu dentro do recomendado. Considerando o limite máximo de energia proveniente de carboidratos $(65,0 \%)$ foram identificadas crianças, em ambas as faixas etárias, $17,1 \%$ e $11,1 \%$, respectivamente, ultrapassando esse limite. Essa diferença é biologicamente significativa, pois reflete o não prolongamento do aleitamento materno até o final do segundo ano de vida, com precoce e inadequada introdução de alimentos da família.

A alimentação complementar das crianças menores de dois anos, em países em desenvolvimento, tem, freqüentemente, focalizado os aspectos de adequação nutricional. Embora se saiba que as crianças no segundo e terceiro anos de vida apresentam maior estabilidade no crescimento, devido à diminuição da velocidade de ganho de peso e estatura, condicionando perda de apetite, assim mesmo a alimentação deve ser capaz de suprir as necessidades de energia, macronutrientes e micronutrientes, de maneira equilibrada ${ }^{26}$.

Com relação ao consumo de energia proveniente da ingestão de proteínas, a maior parte das crianças, de 12 a 23 e 24 a 35 meses de idade, $(96,4 \%$ e $96,8 \%)$ encontrava-se nos limites recomendados da AMDR (5,0\% - 20,0\%), embora 3,6\% de crianças de 12 a 23 meses apresentassem ingestão abaixo do limite mínimo recomendado.

Conforme os resultados encontrados, as crianças deste estudo apresentaram risco de inadequação para micronutrientes essenciais, a exemplo do zinco, vitamina $A$, vitamina $C$ e ferro (Figura 1).

Embora se possa apenas constatar que o risco de inadequação está presente entre as crianças pesquisadas, a deficiência de micronutrientes, como a vitamina A, o ferro e o zinco, comprometem o crescimento e desenvolvimento normais das crianças e diminui a resistência às doenças, especialmente às infecções. 


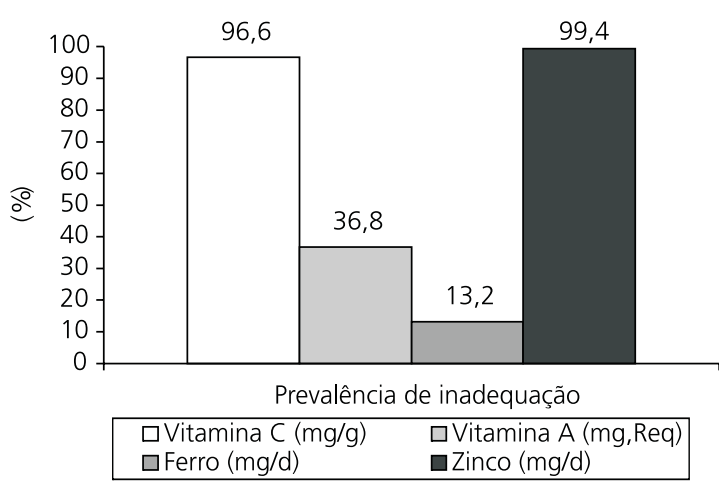

Figura 1. Ingestão de micronutrientes pelas crianças. Viçosa, 2004

Alguns estudos reconhecem que as deficiências de micronutrientes são, ainda, os maiores problemas de saúde pública, com conseqüências diretas sobre o estado de saúde infantil. A deficiência de vitamina A pode aumentar as taxas de morbidade e mortalidade por diarréia e sarampo, a deficiência de zinco comprometer o funcionamento do sistema imunológico, bem como interferir no crescimento e desenvolvimento, uma vez que é um mineral essencial para este fim. A deficiência de ferro é considerada a carência nutricional mais prevalente em todo o mundo, especialmente nos países em desenvolvimento, afetando lactentes, pré-escolares, adolescentes e gestantes. Quando essas deficiências se manifestam nos primeiros dois anos de vida têm sido largamente associadas ao retardo de crescimento, retardo do desenvolvimento psicomotor e diminuição da capacidade de aprendizagem 2,27,28.

Buscando associar o consumo alimentar ao estado nutricional, verificaram-se algumas associações e fatores de risco entre estas variáveis. Com relação ao consumo de ferro (Tabela 2), os resultados indicam que há associação estatisticamente significante entre a ocorrência de déficit nutricional (peso/idade e estatura/idade) e consumo inadequado do referido mineral $(p<0,05)$. Pelo valor da Odds Ratio (Tabela 2), os resultados indicam que crianças com consumo inadequado de ferro apresentaram maior risco de déficit de peso e estatura para a idade.

Tabela 2. Associação entre ocorrência de desnutrição e risco nutricional em crianças com consumo inadequado de ferro. Viçosa, 2004.

\begin{tabular}{|c|c|c|c|c|}
\hline Índices antropométricos & Inadequação (\%) & OR (IC 95\%) & $\chi^{2}$ & Valor de $p$ \\
\hline $\mathrm{P} / \mathrm{I}$ & & 2,84 & & \\
\hline$(\leq-2<-1)$ & 9,8 & $(1,13<\mathrm{OR}<7,25)$ & 6,14 & $p=0,01$ * \\
\hline $\mathrm{P} / \mathrm{E}$ & & 2,18 & & \\
\hline$(\leq-2<-1)$ & 5,7 & $(0,80<O R<5,93)$ & 2,95 & $p=0,08$ \\
\hline$E / l$ & & 3,37 & & \\
\hline$(\leq-2<-1)$ & 6,9 & $(1,37<O R<10,27)$ & 8,67 & $p=0,01$ * \\
\hline
\end{tabular}

${ }^{*} p<0,05 . \mathrm{P} / \mathrm{l}=$ peso/idade; $\mathrm{P} / \mathrm{E}=$ peso/estatura; $\mathrm{E} / \mathrm{l}=$ estatura/idade.

Tabela 3. Associação entre ocorrência de desnutrição e risco nutricional em crianças com consumo inadequado de retinol. Viçosa, 2004.

\begin{tabular}{|c|c|c|c|c|}
\hline Índices antropométricos & Inadequação (\%) & OR (IC 95\%) & $\chi^{2}$ & Valor de $p$ \\
\hline $\mathrm{P} / \mathrm{l}$ & & 1,85 & & \\
\hline$(\leq-2<-1)$ & 19,0 & $(0,95<0 R<3,63)$ & 3,77 & $p=0,05$ \\
\hline $\mathrm{P} / \mathrm{E}$ & & 1,20 & & \\
\hline$(\leq-2<-1)$ & 12,1 & $(0,58<0 R<2,46)$ & 0,29 & $p=0,58$ \\
\hline$E / l$ & & 2,21 & & \\
\hline$(\leq-2<-1)$ & 14,4 & $(1,07<O R<4,59)$ & 5,44 & $p=0,01$ * \\
\hline
\end{tabular}

${ }^{*} p<0,05$. 
Observou-se uma associação estatisticamente significante entre desnutrição e risco nutricional das crianças estudadas e o consumo inadequado de retinol, apenas para o índice estatura/ idade $(p<0,05)$, mostrando que a inadequação de retinol se constituiu em fator de risco para a ocorrência de déficit estatural (Tabela 3).

Analisando-se o estado nutricional pelo índice antropométrico peso/idade (Figura 2) verificou-se que $13,2 \%$ das crianças estudadas estavam desnutridas, 28,7\% encontravam-se em risco nutricional, 50,0\% estavam eutróficas, 7,5\% encontravam-se em risco de sobrepeso e apenas 0,6\% apresentou sobrepeso.

Ainda na Figura 2, o estado nutricional avaliado pelo índice peso/estatura, mostrou 13,8\% das crianças desnutridas, $21,3 \%$ em risco nutricional, 57,5\% encontravam-se eutróficas, $5,7 \%$ em risco de sobrepeso e 1,7\% apresentaram sobrepeso.

Analisando o estado nutricional pelo índice estatura/idade verificou-se que, apesar da maioria das crianças (75,9\%) apresentarem-se eutróficas, uma proporção significativa estava em risco nutricional.

Com relação à prevalência de desnutrição, os resultados são bem inferiores aos encontrados por Lima ${ }^{29}$ no ano de 2001, que encontrou prevalências de desnutrição, pelo índice peso/idade, de $31,0 \%$ e $22,1 \%$ e pelo índice de estatura/idade

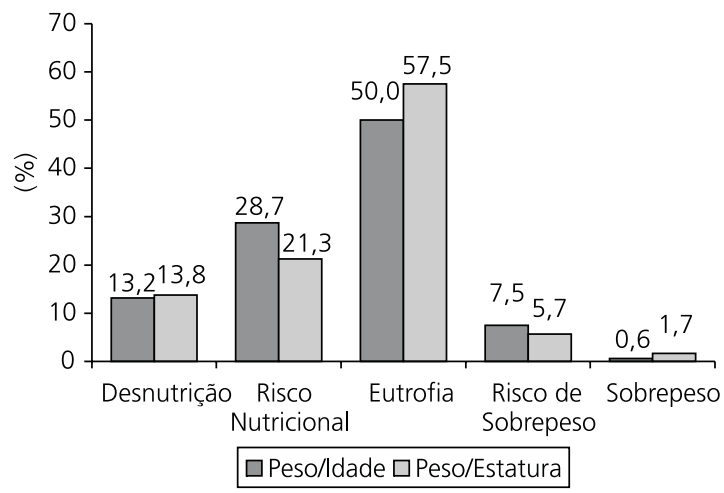

Figura 2. Estado nutricional das crianças, de acordo com os índices peso/idade e peso/estatura. Viçosa, 2004
$27,6 \%$ e $8,6 \%$, nas faixas etárias de $12 \%$ a 24 e $24 \%$ a 36 meses de idade, respectivamente, ao avaliar a evolução do estado nutricional de crianças menores de 5 anos de Viçosa (MG). Entretanto, os resultados deste estudo revelaram que os déficits nutricionais ainda representam um problema importante entre as crianças de $12 \%$ a 36 meses de idade do município de Viçosa e, embora estas prevalências sejam consideradas pela Organização Mundial da Saúde ${ }^{30}$ como de baixa magnitude (10,0\% a 19,9\%), encontram-se ainda mais elevadas que a média verificada em estudos brasileiros $(10,7 \%)^{31}$, pela Pesquisa Nacional sobre Saúde e Nutrição (PNSN) (6,7\%), em 198932, e pela Pesquisa Nacional sobre Demografica e Saúde (PNDS) (5,7\%), em 199633, segundo o índice peso/idade.

Para todos os índices antropométricos avaliados, metade ou mais das crianças encontravam-se eutróficas. No entanto, as elevadas prevalências de crianças em risco nutricional para os índices estatura/idade e peso/idade, sobretudo entre as crianças de 24 a 35 meses de idade, mostram que, historicamente, essas crianças não vêm obtendo os meios necessários para promover adequadamente seu crescimento, podendo refletir, também, as condições vividas no momento atual, como a ocorrência de morbidades (diarréia, pneumonia, infecções da garganta, olhos e nariz) relatadas pelas mães ou responsáveis pela criança, no momento da avaliação.

As prevalências de risco de sobrepeso encontradas neste estudo pelos índices peso/idade e peso/estatura, evidenciam a preocupação mundial de que excesso de peso é um problema de saúde na infância e pode persistir pela vida adulta, fase na qual a morbidade e a mortalidade irão se manifestar com maior intensidade.

As prevalências de sobrepeso, pelo índice peso/idade e peso/estatura foram inferiores ao esperado para uma população saudável $(2,3 \%)$, mas devem se constituir em alerta para futuro risco de obesidade na população estudada ${ }^{22}$. 


\section{O N CLUSÃO}

Considerando a variabilidade referente ao consumo alimentar das crianças pode-se concluir que, apesar do fornecimento de energia por meio da dieta ter excedido a recomendação para a grande maioria das crianças estudadas, foram observadas elevadas prevalências de inadequação para a maior parte dos micronutrientes, a exemplo das vitaminas $\mathrm{A}$ e $\mathrm{C}$ e minerais ferro e zinco, essenciais para prevenir a morbimortalidade nos primeiros anos de vida. Uma significante proporção de crianças encontrava-se fora dos limites de distribuição aceitável de energia proveniente dos macronutrientes, o que, possivelmente, expõe esse grupo aos efeitos e conseqüências adversos para a saúde e nutrição. Ao relacionar o consumo alimentar ao estado nutricional, identificaram-se associações estatisticamente significantes entre inadequação de ferro e retinol e os déficits nutricionais.

A situação alimentar avaliada contribui para deteriorar a saúde e o estado nutricional das crianças, sendo recomendável a realização continuada de estudos de consumo alimentar nessa população, para conhecer melhor a sua prática alimentar.

Especialmente entre as crianças menores de 24 meses de idade, reforço na vigilância alimentar e nutricional, orientação nutricional adequada, com ênfase no estímulo ao prolongamento do aleitamento materno, fortalecimento dos atuais programas de suplementação alimentar e planejamento de mensagens educativas de saúde pública, deverão ser estratégias continuamente estimuladas para prevenir distúrbios alimentares decorrentes do consumo alimentar excessivo ou deficiente.

Enfim, na análise dos dados de consumo de novos estudos, recomenda-se a utilização dos valores de referência Dietary Reference Intakes (DRI), do Instituto de Medicina (Institute of Medicine/Food and Nutrition Board). As DRI, como são nominadas, constituem um grupo de quatro novas recomendações de valores de ingestão representando um importante avanço no modo de analisar e interpretar a ingestão dietética. Esse aspecto é relevante, dada a dificuldade de identificar trabalhos similares, em termos de método de análise, que permitam comparar os dados.

\section{A GRADECIMENTOS}

Os autores agradecem a preciosa colaboração da nutricionista Poliana Cardoso Martins; às mães e suas crianças, pela participação.

\section{REFERÊ NCIAS}

1. Margetts BM, Nelson M. Design Concepts in Nutritional Epidemiology. 2nd ed. New York: Oxford University Press; 1997.

2. Silva DG, Franceschini SCC, Priore SE, Ribeiro SMR, Szarfarc SC, Souza SB, et al. Anemia ferropriva em crianças de 6 a 12 meses atendidas na rede pública de saúde do município de Viçosa, Minas Gerais. Rev Nutr. 2002; 15(3):301-8.

3. Miranda AS, Franceschini SCC, Priore SE, Euclydes MP, Araújo RMA, Ribeiro SMR, et al. Anemia ferropriva e estado nutricional de crianças com idade de 12 a 60 meses do município de Viçosa, MG. Rev Nutr. 2003; 16(2):163-9.

4. Rotenberg S, De Vargas S. Práticas alimentares e o cuidado com a saúde: da alimentação da criança à alimentação da família. Rev Bras Saúde Matern Infant. 2004; 4(1):85-94.

5. Castro TG, Campos FM, Priore SE, Coelho FMG, Campos MTFS, Franceschini SCC, et al. Saúde e nutrição de crianças de 0 a 60 meses de um assentamento de reforma agrária, Vale do Rio Doce, MG, Brasil. Rev Nutr. 2004; 17(2):167-76.

6. Post $C L$, Victora CG, Barros AJD. Baixa prevalência de déficit de peso para estatura: comparação de crianças brasileiras com e sem déficit estatural. Rev Saúde Pública. 1999; 33(6):575-85.

7. Oliveira SP, Thébaud-Mony A. Estudo do consumo alimentar: em busca de uma abordagem multidisciplinar. Rev Saúde Pública. 1997; 31(2):201-8.

8. Bonomo E. Como medir a ingestão alimentar? In: Dutra de Oliveira JE. Obesidade e anemia carencial na adolescência. São Paulo: Instituto Danone; 2000. 
9. Cintra IP, von Der Heyde Med, Schimitz BAS, Franceschini SCC, Taddei JA, Sigulem DM. Métodos de inquéritos dietéticos. Cad Nutr Soc Bras Alim Nutr. 1997; 13:11-23.

10. Sigulem DM, Devincenzi UM, Lessa AC. Diagnóstico do estado nutricional da criança e do adolescente. J Pediatria. 2000; 76(Supl 3): S275-84.

11. Sales RL, Eckhard VF, Costa NMB, Silva MMS, Euclydes MP, Coelho AIM. Desenvolvimento e validação de instrumentos para avaliação da ingestão alimentar de grupos populacionais. In: Anais do VII Simpósio de Iniciação Científica; 1997; Viçosa, Minas Gerais. Viçosa: Universidade Federal de Viçosa; 1997.

12. Pinheiro ABV, Lacerda EMA, Benzecry EH, Gomes MCS, Costa VM. Tabela para avaliação de consumo alimentar em medidas caseiras. Grupo de Pesquisa em Nutrição Materno-Infantil. Rio de Janeiro: UFRJ; 2004. 63p.

13. Sichieri R. Epidemiologia da obesidade. Coleção Saúde e Sociedade. Rio de Janeiro: UERJ; 1998. p.98.

14. Salvo VLMA, Gimeno SGA. Reprodutibilidade e validade do questionário de freqüência de consumo alimentar. Rev Saúde Pública. 2002; 36(4)505-12.

15. Crispim SP, Franceschini SCC, Priore SE, Fisberg RM. Validação de inquéritos dietéticos: uma revisão. Rev Nutrire. 2003; 26(2):127-41.

16. Salvo VLMA, Gimeno SGA. Métodos de investigação do consumo alimentar [entrevista]. [acesso 21 nov 2003]. Disponível em: www.nutricaoempauta.com.br

17. Galeazzi MAM, Meireles AJA, Viana RPT, Zabotto CB, Domene SAM. Registro fotográfico para inquéritos dietéticos: utensílios e porções. Goiânia: Unicamp; 1996.

18. Institute of Medicine. Food and Nutrition Board. Dietary Reference Intakes for Vitamin C, Vitamin E, Selenium and Carotenoids. Washington (DC): National Academy Press; 2000.

19. Institute of Medicine. Food and Nutrition Board. Dietary Reference Intakes for Vitamin A, Vitamin K, Arsenic, Boron, Chromium, Copper, lodine, Iron, Manganess, Molybdenum, Nickel, Vanadium, and Zinc. Washington (DC): National Academy Press; 2001.

20. Institute of Medicine. Food and Nutrition Board. Dietary Reference Intakes for Energy, Carbohydrate, Fiber, Fat, Fatty Acids, Cholesterol, Protein, and Amino Acids. Washington (DC): National Academy Press; 2002.
21. Organización Mundial de la Salud. Medición del cambio del estado nutricional. Ginebra; 1983.

22. CDC Growth Charts for the United States. Methods Development Vital Health Statistic Series. 2000; 11(246):1-190.

23. Institute of Medicine. Dietary Reference Intakes: applications in dietary assessment. Washington (DC): National Academy Press; 2002.

24. Barquera S, Rivera JA, Safdie M, Flores M, Campos-Nonato I, Campirano F. Energy and nutrient intake in preschool and school age Mexican children: National Nutrition Survey 1999. Salud Publica México. 2003; 45(Supl 4):S540-50.

25. Devaney B, KALB L, Zavitsky-Novak T, Clusen N, Ziegler P. Feeding infants and toddlers study: overview of the study design. J Am Diet Assoc. 2004; 104(Suppl 1):S8-S13.

26. Philippi ST, Cruz ATTR, Colucci ACA. Pirâmide alimentar para crianças de 2 a 3 anos. Rev Nutr. 2003; 16(1):5-9.

27. Fundo das Nações Unidas para a Infância. Relatório da Situação da Infância Brasileira. Brasília: Unicef; 2001.

28. Osório MM. Fatores determinantes da anemia em crianças. J Pediat 2002; 72(4):269-78.

29. Lima NMML. Evolução do estado nutricional de crianças menores de 5 anos, após Implantação do sistema de vigilância alimentar e nutricional no município de Viçosa, MG [monografia de especialização]. Viçosa: Universidade Federal de Viçosa; 2002.

30. World Health Organization. Global database on child growth and malnutrition. Program of Nutrition Family and Reproductive Health. Geneve; $1997 a$.

31. Victora CG, Gigante DP, Barros AJD, Monteiro CA, Onis M. Estimativa da prevalência de déficit de altura/idade a partir da prevalência de déficit de peso/idade em crianças brasileiras. Rev Saúde Pública. 1998; 32(4):321-7.

32. Brasil. Pesquisa Nacional Sobre Saúde e Nutrição. Brasília: Ministério da Saúde; 1986.

33. Brasil. Pesquisa Nacional de sobre Demografia e Saúde. Indicadores e dados básicos, 1998. Brasília: Benfam; 1999. 